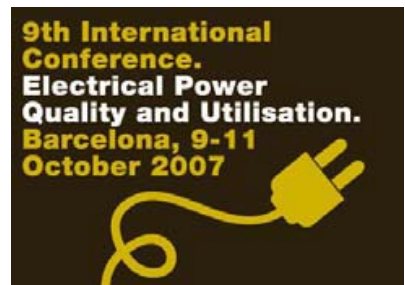

\title{
Ant Colony Solution to Optimal Transformer Sizing Problem
}

\author{
Elefterios I. Amoiralis ${ }^{1}$, Marina A. Tsili ${ }^{2}$, Pavlos S. Georgilakis ${ }^{1}$, and Antonios G. Kladas ${ }^{2}$ \\ ${ }^{1}$ Department of Production Engineering and Management \\ Technical University of Crete \\ Chania, Greece \\ eamir@tee.gr \\ pgeorg@dpem.tuc.gr \\ ${ }^{2}$ Faculty of Electrical \& Computer Engineering \\ National Technical University of Athens \\ Athens, Greece \\ mtsili@central.ntua.gr \\ kladasel@central.ntua.gr
}

\begin{abstract}
This paper proposes a non-deterministic methodology, based on ant colony optimization, for the optimal choice of transformer sizes to serve a forecasted load. This methodology is properly introduced to the solution of the optimal transformer sizing problem, taking into account the constraints imposed by the load to be served by the transformer throughout its life time and the possible thermal overloading. The possibility to upgrade the transformer size one or more times throughout the study period results to different sizing paths, and ant colony optimization is applied in order to determine the least cost path, taking into account the transformer capital cost as well as the energy loss cost during the study period. The results of the proposed methodology demonstrate the benefits of its application in comparison to simplified sizing strategies.
\end{abstract}

Keywords-component; transformers; Optimal Transformer Sizing; Ant Colony Optimization; thermal loading; energy loss cost;

\section{INTRODUCTION}

Due to the large amount of installed distribution transformers in power systems, the impact of their proper sizing, also referred as kVA sizing, is particularly crucial to their design and can result to significant economic benefits to electric utilities and customers. There are several factors involved in the process of sizing a transformer, e.g. expected future growth of the load to be served, installation altitude, ambient temperature, insulation, number of phases, efficiency, losses, capital cost throughout the transformer commission time, cost of the energy, and interest rate. During the solution of the transformer sizing problem, careful consideration must be given to the fact that the possible transformer capacities are discrete instead of continuous and that the option to upgrade the transformer size one or more times throughout the study period can result to different sizing strategies that have to be considered as well.

It is therefore clear that the optimal choice of the transformer size cannot be straightforward, by installing a transformer of sufficient capacity to meet the load demand in the final year of the study period, a strategy usually adopted by electric utilities. On the other hand, it must be treated as a constrained minimization problem, taking into account all possible sizing strategies. Deterministic optimization methods may be used for the solution of this class of problems, such as dynamic programming [1] or integer programming [2]. However, the large quantity of transformers involved in the electric utility distribution system makes the transformer sizing a difficult combinatorial optimization problem, since the space of possible solutions is huge. That is why stochastic optimization methods may prove to provide more robust solutions. The use of such methods in transformer sizing is not encountered in the technical literature or is partially included in the analysis, e.g. as a tool to forecast the loads to be served [3].

In this paper, the Optimal Transformer Sizing (OTS) problem is solved by means of the heuristic Ant System method using the Elitist strategy, called Elitist Ant System (EAS). EAS belongs to the family of Ant Colony Optimization (ACO) algorithms. Dorigo has proposed the first EAS in his Ph.D. thesis [4] and later on in [5][6]. The EAS is a biologically inspired meta-heuristics method in which a colony of artificial ants cooperates in finding good solutions to difficult discrete optimization problems, such as the OTS problem. Cooperation is the key design component of ACO algorithms: the choice to allocate the computational resources to a set of relatively simple agents (artificial ants) that communicate indirectly by stigmergy [7], i.e. by indirect 
communication mediated by the environment. In other words, a set of artificial ants cooperate in dealing with a problem by exchanging information via pheromones deposited on a graph. In the literature, ACO algorithms can be applied to solve a variety of well-known combinatorial optimization problems, such as routing problem [8], assignment problem [9], scheduling problem [10], and subset [11] problems. In power systems, ACO algorithms have been applied to solve the optimum generation scheduling problem [12][13], and the optimum switch relocation and network reconfiguration problems for distribution systems [14][15][16]. More details on ACO implementation in the solution of other problems are described in [17].

The present paper introduces the use of EAS in the solution of the OTS problem in case of three-phase, oil-immersed, selfcooled (ONAN) distribution transformers. This method is particularly suitable for the considered combinatorial optimization problem, providing an enhanced performance in the location of the optimum, compared to deterministic methods. The OTS problem is solved as a constrained optimization problem, following the procedure described in Section II. The most important constraint is the load to be served by the transformer throughout its life cycle, as well as the respective transformer thermal loading, evaluated through detailed calculation of the winding temperature variation, as described in Section III. The calculation takes into account the rest of the constraints that have to be met, including insulation aging throughout the transformer life cycle for the specified installation altitude conditions. The objective function to be minimized includes the transformer capital cost as well as the energy loss cost (Section IV). The EAS method (Section V) is adapted to the considered problem and the results of its implementation (Section VI) demonstrate its suitability for the solution of OTS and the benefits from its application in comparison to simplified transformer sizing approaches.

\section{OVERVIEW OF THE Proposed Method}

The optimal transformer sizing problem consists in finding the proper transformer capacities and technical characteristics so that the overall cost over the transformer's life cycle can be minimized and the peak loading condition can be met [1].

The process of the optimal transformer sizing is realized through the following steps:

- Collection of data concerning the special characteristics of the load to be served by the transformer to be installed. These data consist of the typical daily load curve and the expected load growth rate over the transformer lifetime.

- Definition of the number of years of the study period.

- Selection of possible candidate transformer sizes according to tables of standard transformer sizes, although nonstandard sizes can be also considered.

- Determination of the feasible number of years that each potential transformer can serve the examined load, based on the thermal calculations presented in Section III.

- Determination of the possible transformer sizing strategies throughout the study period, consisting of combinations of the potential transformer capacities and the years that they may operate in order to serve the considered load.

- Calculation of the energy loss cost for each transformer (through the method presented in Section IV) for the periods defined by the strategies of the previous step.

- Creation of the graph with all possible combinations of transformer sizes and years of replacement, for the solution of the OTS problem with the use of EAS, according to the procedure described in Section V. The cost of each path consists of the energy loss cost of the installed transformer $S_{i}$ for the period of years of operation until the upgrade to a larger capacity and the bid price of the new transformer $S_{j}$ to be installed.

- Selection of the least cost transformer sizing path, with the use of EAS. This path corresponds to the optimal transformer sizing strategy.

\section{CAlculation OF TRANSFORMER Thermal LOADING}

The calculation is implemented according to the guidelines imposed by the IEEE Standard C57.91-1995 (R2002), [18]. The main equations used in these calculations are described in the followings (all temperatures are expressed in ${ }^{\circ} \mathrm{C}$ ).

\section{A. Top-oil temperature calculation}

The top-oil temperature rise $\Delta \Theta_{T O}$ at a time after a step load change is given by the following equation:

$$
\Delta \Theta_{T O}=\left(\Delta \Theta_{T O, U}-\Delta \Theta_{T O, i}\right) \cdot\left(1-e^{\frac{-1}{\tau_{T O}}}\right)+\Delta \Theta_{T O, i}
$$

where $\Delta \Theta_{T O, i}$ and $\Delta \Theta_{T O, U}$ are the initial and ultimate top-oil rise over ambient temperature during the considered time period, while $\tau_{T O}$ is the oil time constant (in hours) for the considered load, deriving from the value of the time constant at the rated load $\tau_{T O, R}$ [18]. A typical value of $\tau_{T O, R}$ equal to 2.5 hours can be considered for distribution transformers larger than 200kVA [19].

The initial top-oil rise over ambient temperature $\Delta \Theta_{T O, i}$ is equal to the value of $\Delta \Theta_{T O}$ calculated at the previous interval of the considered load cycle. The ultimate top-oil rise over ambient temperature is calculated with the use of the top-oil rise over ambient temperature at rated load $\Delta \Theta_{T O, R}$ (equal to $60^{\circ} \mathrm{C}$ for the considered distribution transformers), the ratio $K$ of the load at the considered interval to the rated load and the ratio $R$ of the load loss at rated load to no-load loss:

$$
\Delta \Theta_{T O, U}=\Delta \Theta_{T O, R} \cdot\left(\frac{K^{2} \cdot R+1}{R+1}\right)^{n}
$$


For the first interval of the studied load cycle, an initial value of $\Delta \Theta_{T O, i}$ equal to zero may be arbitrarily chosen. After completing the above calculations for all the intervals of the load cycle, the final $\Delta \Theta_{T O, U}$ of the cycle can be used as the new value of $\Delta \Theta_{T O, i}$ of the first interval and the process may be repeated to obtain stable temperature profiles.

\section{B. Winding hottest spot temperature calculation}

The winding hottest spot temperature rise over top-oil temperature $\Delta \Theta_{H}$ at a time after a step load change is given by the following equation:

$$
\Delta \Theta_{H}=\left(\Delta \Theta_{H, U}-\Delta \Theta_{H, i}\right) \cdot\left(1-e^{\frac{-1}{\tau_{w}}}\right)+\Delta \Theta_{H, i}
$$

where $\Delta \Theta_{H, i}$ and $\Delta \Theta_{H, U}$ represent the initial and ultimate winding hottest spot temperature rise over top-oil temperature during the considered time period and $\tau_{w}$ stands for the winding time constant at hot spot location (in hours). The typical value of $\tau_{w}$ is less than 0.1 hours [20], thus (3) may be simplified to the following equation:

$$
\Delta \Theta_{H}=\Delta \Theta_{H, U}
$$

The ultimate winding hottest spot temperature rise over topoil temperature is given by:

$$
\Delta \Theta_{H, U}=\Delta \Theta_{H, R} \cdot K^{2 m}
$$

where $\Delta \Theta_{H, R}$ is the winding hottest spot temperature rise over top-oil temperature at the rated load (a value of $15^{\circ} \mathrm{C}$ is suggested by the standard, when the actual test values are not available) and $m$ is an empirical factor, equal to 0.8 for selfcooled transformers.

Finally, the winding hottest spot temperature at the considered interval is the sum of $\Delta \Theta_{T O}, \Delta \Theta_{H}$ and the average ambient temperature $\Theta_{A}$ during the cycle:

$$
\Theta_{H}=\Theta_{A}+\Delta \Theta_{T O}+\Delta \Theta_{H}
$$

\section{Insulation aging}

Although the IEEE standard specifies that operation at hottest spot temperature above $140^{\circ} \mathrm{C}$ results to potential risks for the transformer integrity, this value must not be considered as the maximum one during the calculations carried out for the determination of the transformer loading limits. This is due to the fact that thermal aging is a cumulative process, therefore operation above the rating should be examined in conjunction with its consequences upon the normal life expectancy of the transformer. For the study of the present paper, a maximum hot-spot temperature of $120^{\circ} \mathrm{C}$ has been chosen, based on the relative aging rate of the insulation in the transformer [19].

\section{Overloading capability}

In order to determine the transformer loading limits, the calculation of the hottest spot temperature is repeated for each year of the study period, at an hourly basis, according to the daily load curve. In order to remain on the safe side, the peak load curve of the considered year is used in the calculations. The yearly load growth rate $r$ is taken into account for the derivation of the per unit load $K_{t}^{j}$ of hour $t$ at year $j$ of the study according to the per unit load $K_{t}^{0}$ of hour $t$ at year 0 :

$$
K_{t}^{j}=K_{t}^{0} \cdot(1+r)^{j}
$$

Fig. 1 shows the winding hottest spot temperature variation for three distribution transformers of rated capacity 250, 400 and $630 \mathrm{kVA}$ and ratio $R$ of the load loss at rated load to noload loss equal to $5.23,4.73$ and 7.11 , respectively, serving a residential load of initial peak value equal to $230 \mathrm{kVA}$, at the $10^{\text {th }}$ year of the study period, for a load growth rate of $3.7 \%$. The scale of the left axis in Fig.1 corresponds to the values of the load curve $K_{t}^{j}$ (expressed in kVA, since the per unit values are different on the basis of the rated capacity of each transformer), illustrated as bar diagram. The scale of the right axis in Fig.1 indicates the winding hottest spot variation, in ${ }^{\circ} \mathrm{C}$ (corresponding to the three transformer ratings of Fig.1). The thermal calculation is based on the load curve of summer, with the use of an ambient temperature equal to $40^{\circ} \mathrm{C}$. As can be observed from Fig. 1, the $250 \mathrm{kVA}$ transformer overcomes the limit of $120^{\circ} \mathrm{C}$ so it is not suitable to serve the load at the $10^{\text {th }}$ year of the study period.

\section{Calculation of Transformer Energy Loss Cost}

The calculation of transformer energy loss cost for each period of the potential sizing paths is realized with the use of the energy corresponding to the transformer no load losses $(N L L) E_{N L L}^{k}$ (in $\mathrm{kWh}$ ) for a period of $k$ years of operation and the energy corresponding to the load losses $(L L), E_{L L}^{k}$ (in $\mathrm{kWh}$ ) for a period of $k$ years of operation. These energies are calculated according to (8) and (9):

$$
E_{N L L}^{k}=N L L \cdot H P Y \cdot k
$$

$$
E_{L L}^{k}=L L \cdot l_{f}^{2} \cdot H P Y \cdot k
$$

where $H P Y$ is the number of hours per year, equal to 8760 and $l_{f}$ is the load factor, i.e. the mean transformer loading over its lifetime. The cost of total energy corresponding to the transformer $N L L$ for the period of $k$ years $C_{N L L}^{k}$ (in $€$ ) and the 
cost of energy corresponding to the transformer $L L$ over a period of $k$ years $C_{L L}^{k}$ (in $€$ ) derive as follows:

$$
\begin{gathered}
C_{N L L}^{k}=E_{N L L}^{k} \cdot C Y E C \\
C_{L L}^{k}=E_{L L}^{k} \cdot C Y E C
\end{gathered}
$$

where $C Y E C$ denotes the present value of the energy cost (in $€ / \mathrm{kWh}$ ). Finally, the total cost of the transformer energy loss $E_{L}^{k}$ for the period of $k$ years of the study is given by:

$$
E_{L}^{k}=C_{N L L}^{k}+C_{L L}^{k}
$$

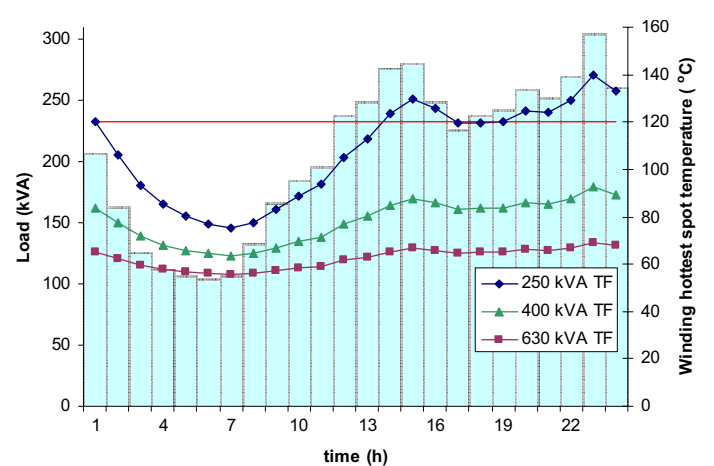

Figure 1. Winding hottest spot temperature variation of a 250, 400 and 630 $\mathrm{kVA}$ transformer (TF) during a daily load cycle.

\section{Elitist Ant System Method}

\section{A. Mechanism of EAS algorithm}

The EAS is an evolutionary computation optimization method based on ants' collective problem solving ability. This global stochastic search method is inspired by the ability of a colony of ants to identify the shortest route between the nest and a food source, without using visual cues.

The operation mode of EAS algorithm is as follows: the artificial ants of the colony move, concurrently and asynchronously, through adjacent states of a problem, which can be represented in the form of a weighted graph. This movement is made according to a transition rule, called random proportional rule, through a stochastic mechanism. When ant $k$ is in node $i$ and has so far constructed the partial solution $s^{p}$, the probability of going to node $j$ is given by:

$$
p_{i j}^{k}=\left\{\begin{array}{c}
\frac{\tau_{i j}^{\alpha}+n_{i j}^{\beta}}{\sum_{c_{i l} \in N\left(s^{p}\right)} \tau_{i l}^{\alpha}+n_{i l}^{\beta}}, \text { if } c_{i j} \in N\left(s^{p}\right) \\
0 \quad, \text { otherwise }
\end{array}\right.
$$

where $N\left(s^{p}\right)$ is the set of feasible nodes when being in node $i$, i.e. edges $(i, l)$ where $l$ is the node not yet visited by the ant $k$. The parameters $\alpha$ and $\beta$ control the relative importance of the pheromone versus the heuristic information value $\eta_{i j}$, given by:

$$
n_{i j}=\frac{1}{d_{i j}}
$$

where $d_{i j}$ is the weight of each edge. Regarding parameters $\alpha$ and $\beta$, their role is as follows: if $\alpha=0$, those nodes with better heuristic preference have a higher probability of being selected. However, if $\beta=0$, only the pheromone trails are considered to guide the constructive process, which can cause a quick stagnation, i.e. a situation where the pheromone trails associated with some transitions are significantly higher than the remainder, thus making the ants build the same solutions. Hence, there is a need to establish a proper balance between the importance of heuristic and pheromone trail information.

Individual ants contribute their own knowledge to other ants in the colony by depositing pheromones, which act as a chemical "markers" along the paths they traverse. Through indirect communication with other ants via foraging behavior, a colony of ants can establish the shortest path between the nest and the food source over time with a positive feedback loop known as stigmergy. As individual ants traverse a path, pheromones are deposited along the trail, altering the overall pheromone density. More trips can be made along shorter paths and the resulting increase in pheromone density attracts other ants to these paths. The main characteristic of the EAS technique is that (at each iteration) the pheromone values are updated by all the $k$ ants that have built a solution in the iteration itself. The pheromone $\tau_{i j}$, associated with the edge joining nodes $i$ and $j$, is updated as follows [21]:

$$
\tau_{i j}=(1-\rho) \cdot \tau_{i j}+\sum_{m=1}^{k} \Delta \tau_{i j}^{k}+\varepsilon \cdot \tau_{i j}^{e l i t e}
$$

where $\rho \in(0,1]$ is the evaporation rate, $\mathrm{k}$ is the number of ants, $\varepsilon$ is the number of elitist ants, and $\Delta \tau_{i j}^{k}$ is the quantity of pheromone laid on edge $(i, j)$ by ant $\mathrm{k}$, calculated as follows:

$$
\Delta \tau_{i j}^{k}=\left\{\begin{array}{l}
\frac{Q}{L_{k}}, \text { if ant } k \text { used edge }(i, j) \text { in its tour } \\
0, \text { otherwise }
\end{array}\right.
$$

where $Q$ is a constant for pheromone update, and $L_{k}$ is the length (or the weight of the edge) of the tour constructed by ant $k$. Furthermore, shorter paths will tend to have higher pheromone densities than longer paths since pheromone density decreases over time due to evaporation [5][6]. This shortest path represents the global optimal solution and all the possible paths represent the feasible region of the problem. 


\section{B. OTS implementation using the EAS algorithm}

In this work our interest lies in finding the optimum choice of distribution transformers capacity sizing, so as to meet the load demand for all the years of the study period. To achieve so, a graph shown in Fig. 2 is constructed, representing the different sizing paths.

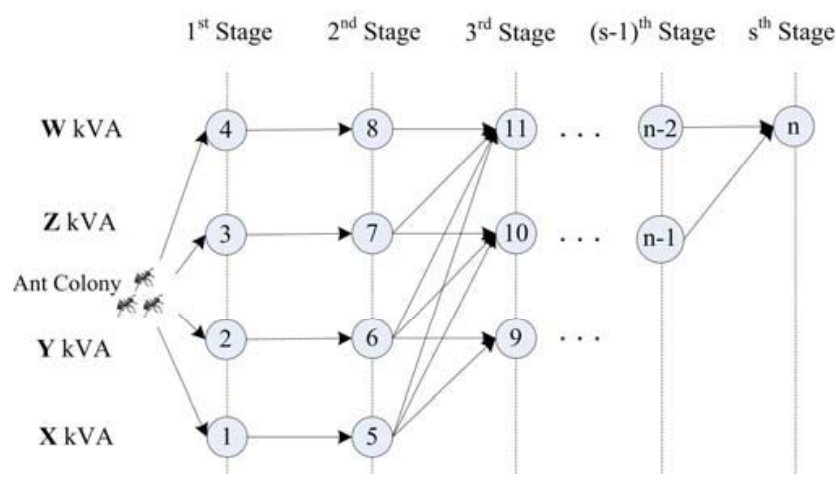

Figure 2. The directed graph used for the OTS problem.

The graph has $s$ stages and each stage indicates a time period (in years) the limits of which are defined by the need to replace one of the considered transformer sizes due to violation of its thermal loading limits. Therefore, stage $s$ has one node less in comparison with stage $(s-1)$, stage $(s-2)$ has one node less in comparison with stage $(s-1)$, etc. First stage indicates the beginning of the study, comprising number of nodes equal to the number of potential transformers, while $s$ represents the end of the study period (consisting of the largest necessary rated capacity able to serve the load at the final year of the study). Symbols $X, Y, Z, W$ refer to the different rated powers $(X>Y>Z>W)$. It is important to point out that it is meaningless to connect for example node 4 to node 6 due to the fact that it corresponds to installation of a transformer with lower rated power than the one already in operation (only upgrade of the sizes can be considered). Furthermore, the arcs between the nodes are directed from the previous stage to the next one (backward movement is not allowed) since each stage represents a forward step in the time of the study. Node 1 , node 2 , node 3 and node 4 are designated as the source node corresponding to each potential transformer size and node $\mathrm{n}$ is designated as the destination node (Fig. 2). The use of multiple source nodes enables the examination of more potential solutions of the problem. The objective of the colony agents is to find the least-cost path between nodes that belong to first stage and node $\mathrm{n}$ that belongs to $s$-th stage. Three quantities are associated with each arc: the arc weight, the pheromone strength, and the agent learning parameter. Based on these characteristics, the weight of each arc is calculated.

TABLE I. TECHNICAL PARAMETERS AND THERMAL WITHSTAND OF THE TRANSFORMERS USED IN THE SOLUTION OF THE OTS PROBLEM.

\begin{tabular}{ccccc}
\hline $\begin{array}{c}\text { Transformer } \\
\text { size }(\boldsymbol{k} \boldsymbol{V A})\end{array}$ & $\begin{array}{c}\text { Bid price } \\
(\boldsymbol{\epsilon})\end{array}$ & $\begin{array}{c}\boldsymbol{N L L} \\
(\boldsymbol{W})\end{array}$ & $\begin{array}{c}\boldsymbol{L} \boldsymbol{( W )} \\
(\boldsymbol{W})\end{array}$ & $\begin{array}{c}\text { Thermal durability } \\
\text { (years) }\end{array}$ \\
\hline 250 & 6916 & 702 & 3672 & 5 \\
300 & 9274 & 738 & 4186 & 10 \\
400 & 10740 & 991 & 4684 & 18
\end{tabular}

\begin{tabular}{ccccc}
\hline $\begin{array}{c}\text { Transformer } \\
\text { size }(\boldsymbol{k V A})\end{array}$ & $\begin{array}{c}\text { Bid price } \\
(\boldsymbol{\epsilon})\end{array}$ & $\begin{array}{c}\text { NLL } \\
(\boldsymbol{W})\end{array}$ & $\begin{array}{c}\boldsymbol{L} \boldsymbol{( \boldsymbol { W } )} \\
500\end{array}$ & $\begin{array}{c}\text { Thermal durability } \\
\text { (years) }\end{array}$ \\
\hline 630 & 13299 & 1061 & 5771 & 24 \\
& 16264 & 1094 & 7774 & 25
\end{tabular}

\section{RESUlTS AND DISCUSSION}

The proposed method is applied for the optimal choice of the transformer size to serve a residential load of initial peak value equal to $230 \mathrm{kVA}$ with a growth rate equal to $3.7 \%$ for 25 years, resulting to final peak value of $659.65 \mathrm{kVA}$ (at the $25^{\text {th }}$ year of the study). Five ratings are considered, namely 250 , $300,400,500$ and $630 \mathrm{kVA}$. Table I lists the main technical characteristics and the estimated bid price of the considered transformers. Initially, thermal calculation study was carried out in order to find the exact periods where each transformer can meet the load expectations. The calculations described in Section III and illustrated in Fig. 1 were repeated for the five transformers and each year of the study, resulting to the time periods of Table I (indicated as years of thermal durability, i.e. years that the transformer is able to withstand the respective thermal loading). These periods were used to define the stages of the graph of Fig. 3. In order to define the weight of each arc in the graph of Fig. 3, the energy loss cost calculation of each transformer for the studied period was realized (Section IV). Table II lists the cost of each arc, calculated according to the procedure described in Section IV. For instance, the arc cost to transit from node 10 to node 14 is computed as follows: Given that $N L L=1.094 \mathrm{~kW}, L L=7.774 \mathrm{~kW}, H P Y=8760 \mathrm{hr} /$ year, and $k=5$ years, from (9) and (10) we have $E_{N L L}^{5}=9583.4 \mathrm{kWh} / \mathrm{yr}$ and $E_{L L}^{5}=31489.5 \mathrm{kWh} / \mathrm{yr}$. For a value of $\mathrm{CYEC}=0.054 € /$ $\mathrm{kWh}$, we get from (11) and (12), $C_{N L L}^{5}=2588 €$ and $C_{L L}^{5}=8502 €$. Finally, from (13) we get $E_{630}^{5-\text { eear }}=11090 €$ for a 5 -year period of the study.

Fig. 3 illustrates the graph of the OTS problem. We tested several values for each parameter, i.e. $\alpha \in\{0,0.5,1,2,5\}, \beta \in\{0,0.5,1,2,5\}, \rho \in\{0.1,0.3,0.5,0.7,1\}$.

Finally, the optimum path is: $3-8-12-15-19-20$, as it is illustrated in Fig. 3 in bold line, and its cost is $69410 €$. The optimal solution is obtained using $k=20, \alpha=2, \beta=0.5, \rho=0.5$, $Q=2.7$, max iterations $=2000$.

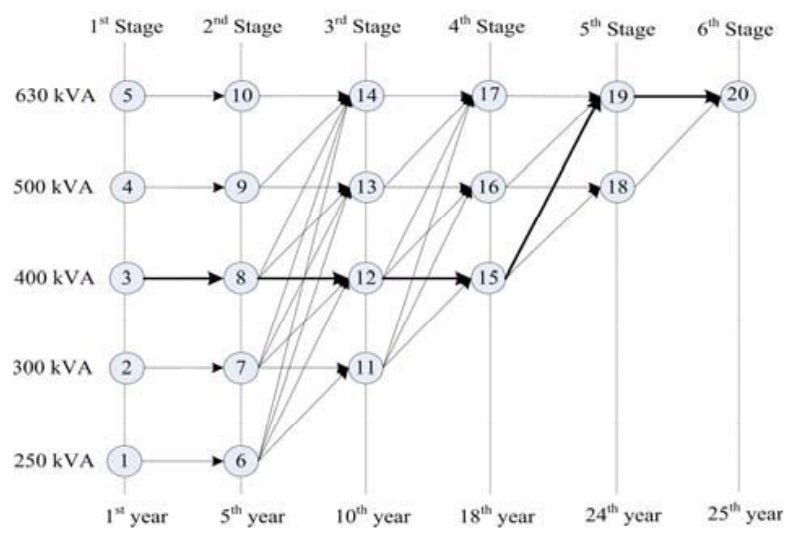

Figure 3. The directed graph used for the proposed OTS problem. 
The cost of the optimal solution provided by the EAS algorithm was compared to a simplified sizing approach, consisting in the selection and installation of the largest necessary capacity, i.e. $630 \mathrm{kVA}$ from the first year of the study. The cost of this solution is equal to the sum of the bid price of the $630 \mathrm{kVA}$ transformer and its energy loss cost for the 25 years of the study, resulting to the value of $71712 €$. This implies that a cost saving of $2302 €$ (or $3.21 \%$ ) is obtained by the proposed ACO method.

TABLE II. COST OF INDICATIVE ARCS IN THE GRAPH OF FIG. 3.

\begin{tabular}{|c|c|c|}
\hline$A r c$ & Cost of the arc & Value (t) \\
\hline $3 \rightarrow 8$ & $E_{400}^{5-\text { year }}+B P_{400}$ & 18207 \\
\hline $8 \rightarrow 12$ & $E_{400}^{5-\text { year }}$ & 7467 \\
\hline $12 \rightarrow 15$ & $E_{400}^{8-\text { year }}$ & 11947 \\
\hline $15 \rightarrow 19$ & $E_{630}^{6-\text { year }}+B P_{630}$ & 29571 \\
\hline $19 \rightarrow 20$ & $E_{630}^{6-\text { year }}$ & 2218 \\
\hline $5 \rightarrow 10$ & $E_{630}^{5-\text { year }}+B P_{630}$ & 27353 \\
\hline $10 \rightarrow 14$ & $E_{630}^{5-\text { year }}$ & 11090 \\
\hline $14 \rightarrow 17$ & $E_{630}^{8-\text { year }}$ & 17744 \\
\hline $17 \rightarrow 19$ & $E_{630}^{6-\text { year }}$ & 13308 \\
\hline $1 \rightarrow 6$ & $E_{250}^{5-\text { year }}+B P_{250}$ & 12592 \\
\hline $6 \rightarrow 11$ & $E_{300}^{5-\text { year }}+B P_{300}$ & 15598 \\
\hline $11 \rightarrow 15$ & $E_{400}^{8-\text { year }}+B P_{400}$ & 22687 \\
\hline $15 \rightarrow 18$ & $E_{500}^{6-\text { year }}+B P_{500}$ & 23884 \\
\hline $18 \rightarrow 20$ & $E_{630}^{6-\text { year }}+B P_{630}$ & 18482 \\
\hline $2 \rightarrow 7$ & $E_{300}^{5-\text { year }}+B P_{300}$ & 15598 \\
\hline $7 \rightarrow 12$ & $E_{400}^{5-\text { year }}+B P_{400}$ & 18207 \\
\hline $12 \rightarrow 16$ & $E_{500}^{8-\text { year }}+B P_{500}$ & 27412 \\
\hline $16 \rightarrow 19$ & $E_{630}^{6-\text { year }}+B P_{630}$ & 29571 \\
\hline
\end{tabular}

VII. CONCLUSIONS

In the present paper, an EAS algorithm was proposed to solve the OTS problem. The EAS algorithm belongs to the ACO methodology. The proposed methodology takes into account the constraints that are imposed when deciding the sizing of a distribution transformer to meet the demands of the expected load to be served, through detailed calculation of its thermal performance throughout the period of the study and accurate representation of the load characteristics. The cost function used in the optimization process includes the transformer energy loss cost as well as its purchasing price. The method was applied for the selection of the optimal size of a distribution transformer (among 5 potential capacities) to serve a load over a period of 25 years. The efficiency of the EAS algorithm relies in converging to the global optimal sizing path, among the numerous possible solutions. Moreover, the the proposed algorithm implies a $3.21 \%$ cost saving in comparison with the simplified sizing strategy usually adopted by electric utilities.

\section{ACKNOWLEDGMENT}

This paper is part of the 03ED045 research project that is co-financed by E.U.-European Social Fund $(75 \%)$ and the Greek Ministry of Development-GSRT (25\%).

\section{REFERENCES}

[1] C.-S. Chen, T.-H. Wu, "Optimal distribution transformer sizing by dynamic programming," Elecrical Power \& Energy Systems, Vol. 20, No. 3, pp. 161-167, 1998.

[2] D. Jovanovic, "Planning of optimal location and sizes of distribution transformers using integer programming," Elecrical Power \& Energy Systems, Vol. 25, pp. 717-723, 2003.

[3] M. Robinson, S. Wallace, D. Woodward, G. Engstrom, "US Navy power transformer sizing requirements using probabilistic analysis," Journal of Ship Production, Vol. 22, No. 4, pp. 212-218, Nov. 2006.

[4] M. Dorigo, "Optimization, learning and natural algorithms," $\mathrm{Ph} . \mathrm{D}$. Thesis (in Italian), Politechnico de Milano, Milan, Italy, 1992.

[5] M. Dorigo, V. Maniezzo, A. Colorni, "Ant System: Optimization by a colony of cooperating agents," IEEE Transactions on Systems, Man, and Cybernetics-Part B, Vol. 26, No. 1, pp. 29-41, 1996.

[6] M. Dorigo, T. Stützle, Ant Colony Optimization, MIT Press, 2004.

[7] S. Goss, S. Aron, J. L. Deneubourg, J. M. Pasteels, "Self-organized shortcuts in the argentine ant," Naturwissenschaften, Vol. 76, 1989.

[8] T. Stützle, H. H. Hoos, "MAX-MIN Ant System," Future Generation Computer Systems, Vol. 16, No. 8, pp. 889-914, 2000.

[9] V. Maniezzo, "Exact and approximate nondeterministic tree-search procedures for the quadratic assignment problem," INFORMS Journal on Computing, Vol. 11, No. 4, pp. 358-369, 1999.

[10] D. Merkle, M. Middendorf, H. Schmeck, "Ant colony optimization for resource-constrained project scheduling," IEEE Transactions on Evolutionary Computation, Vol. 6, No. 4, pp. 333-346, 2002

[11] G. Leguizamon, Z. Michalewicz, "A new version of ant system for subset problems," In Proc. of the 1999 Congress on Evolutionary Computation, Vol. 2, pp. 1459-1464, Washington, USA, 1999.

[12] I. K. Yu, C. S. Chou, Y. H. Song, "Application of the ant colony search algorithm to short-term generation scheduling problem of thermal units," in Proc. Int. Conf. Power Syst. Tech., vol. 1, pp. 552-556, China, 1998.

[13] S. J. Huang, "Enhancement of hydroelectric generation scheduling using ant colony system based optimization approaches," IEEE Trans. Energy Conver., vol. 16, no. 3, pp. 296-301, Sep. 2001.

[14] J. H. Teng, Y.-H. Liu, "A novel ACS-based optimum switch relocation method," IEEE Trans. Power Syst., Vol. 18, No. 1, pp. 113-120, 2003.

[15] Y. J. Jeon, J. C. Kim, S. Y. Yun, K. Y. Lee, “Application of ant colony algorithm for network reconfiguration in distribution systems," in Proc. Symp. Power Plants Power Syst. Control, pp. 266-271, Korea, 2003.

[16] J. G. Vlachogiannis, N. D. Hatziargyriou, K. Y. Lee, "Ant Colony System-Based Algorithm for Constrained Load Flow Problem", IEEE Trans. on Power Systems, Vol. 20, No. 3, pp. 1241-1249, 2005.

[17] C. Blum, "Ant colony optimization: Introduction and recent trends," Physics of Life Reviews, Elsevier, Vol. 2, No. 4, pp.353-373, 2005.

[18] IEEE Guide for Loading Mineral-Oil-Immersed Transformers, IEEE Standard C57.91-1995 (R2002), June 2002.

[19] S.W. Heunis, R. Herman, "A thermal loading guide for residential distribution transformers based on time-variant current load models," IEEE Trans. Power Syst., Vol. 19, No. 3, pp. 1294-1298, Aug. 2004.

[20] K. Schneider, R. Hoad, "Initial transformer sizing for single-phase residential load," IEEE Trans. Power Delivery, Vol. 7, No. 4, pp. 20742081, Oct. 1992.

[21] E. Bonabeau, M. Dorigo, G. Theraulaz, Swarm Intelligence from Natural to Artificial Systems, Oxford University Press, New York, 1999. 